\title{
Association between new-onset anosmia and positive SARS- CoV-2 tests among people accessing outpatient testing in Toronto, Ontario: a retrospective cross-sectional study
}

\author{
Braden O’Neill MD DPhil, Sumeet Kalia MSc, Peter Gill MD DPhil, Susan Hum MSc, \\ Carla Moran-Venegas MSc, Rebecca Stoller MD, Michelle Greiver MD MSc, Payal Agarwal MD, \\ Abirami Kirubarajan MD MSc, Samuel DeKoven MD, David Eisen MD, Andrew Pinto MD MSc, \\ Sheila Dunn MD MSc
}

\section{Abstract}

Background: Reports have suggested that anosmia is strongly associated with SARS-CoV-2 infection, but patients were often asked about this symptom after their diagnosis. This study assessed associations between prospectively reported anosmia and other symptoms related to SARS-CoV-2 infection, and SARS-CoV-2 positivity in community testing centres in Toronto, Ontario.

Methods: We conducted a retrospective cross-sectional study in which data were collected from 2 COVID-19 assessment centres affiliated with 2 hospitals in Toronto, Ontario, from Apr. 5 to Sept. 30, 2020. We included symptomatic profiles of all people who underwent a SARS-CoV-2 test at either clinic within the study period. We used generalized estimating equations to account for repeat visits and to assess associations between anosmia and other symptoms and SARS-CoV-2 positivity.

Results: A total of 83443 SARS-CoV-2 tests were conducted across the 2 sites for 72692 participants during the study period. Of all tests, $1640(2.0 \%)$ were positive; $837(51.0 \%)$ of people who tested positive were asymptomatic. The adjusted odds ratio for the association between anosmia and test positivity was 5.29 (95\% confidence interval $[\mathrm{Cl}] 4.50-6.22)$, with sensitivity of $0.138(95 \% \mathrm{Cl}$ $0.121-0.154)$, specificity of $0.980(95 \% \mathrm{Cl} 0.979-0.981)$, a positive predictive value of $0.120(95 \% \mathrm{Cl} 0.106-0.135)$ and a negative predictive value of $0.983(95 \% \mathrm{Cl} 0.982-0.984)$.

Interpretation: Anosmia had high specificity and a positive predictive value of $12 \%$ for SARS-CoV-2 infection in this community population with low prevalence of SARS-CoV-2 positivity. The presence of anosmia should increase clinical suspicion of SARS-CoV-2 infection, and our findings suggest that people presenting with this symptom should be tested.

OVID-19, caused by infection with SARS-CoV-2, has spread rapidly around the world. As of Nov. 18, 2021, there were more than 255 million cases of COVID-19 and almost 5.2 million deaths worldwide. ${ }^{1}$ There has been a substantial effort to determine specific signs and symptoms predictive of the infection to support screening recommendations or self-isolation to prevent further transmission. ${ }^{2}$ Symptoms of SARS-CoV-2 infection, such as fever, cough and shortness of breath, are nonspecific and common in other respiratory viral infections. ${ }^{3}$ Furthermore, many people with SARS-CoV-2 infection have minimal or no symptoms and are unaware that they may be transmitting the virus. ${ }^{4}$

Anosmia (loss of sense of smell) is a symptom that has received substantial interest, starting with case reports, then media coverage, and followed by large-scale observational studies. $^{5-12}$ Early in the pandemic, the British Rhinological Society suggested that anosmia may be a unique symptom associated with early SARS-CoV-2 infection ${ }^{5}$ and advised anyone with loss of smell to self-isolate. Subsequently, associations between
SARS-CoV-2 infection and anosmia were reported across multiple settings, but these studies have been primarily retrospective, asking people if they experienced these symptoms after they knew their test results, ${ }^{7,11}$ with a high risk of recall bias. A recent systematic review of the diagnostic accuracy of several

Competing interests: Rebecca Stoller declares a stipend as medical lead for the North York General Hospital COVID-19 assessment centre. Michelle Greiver reports a grant to her department from Sanofi, GlaxoSmithKline and Novartis for the development of a severe asthma registry using electronic medical record data. Michelle Greiver is director of UTOPIAN (University of Toronto Practice-Based Research Network) and lead of POPLAR (Primary care Ontario Practice-based Learning and Research Network). No other competing interests were declared.

This article has been peer reviewed.

Correspondence to: Braden O'Neill, braden.oneill @ unityhealth.to CMAJ Open 2021 December 7. DOI:10.9778/cmajo.20210085 
signs and symptoms, including anosmia and COVID-19 diagnosis, reported sensitivity of $28.0 \%$ (95\% confidence interval [CI] $17.7 \%-41.3 \%$ ) and specificity of $93.4 \%$ (95\% CI $88.3 \%-$ $96.4 \%$ ) for anosmia but noted a lack of prospective studies on this association. ${ }^{12}$ Furthermore, anosmia is common: at any time, $3 \%-20 \%$ of the general population ${ }^{13,14}$ may develop "olfactory dysfunction," of which anosmia is a subset. Up to $40 \%$ of anosmia cases are postviral, and coronaviruses are thought to cause $10 \%-15 \%$ of these cases. ${ }^{13}$

"Test and trace" approaches have been adopted to control the spread of SARS-CoV-2. ${ }^{15}$ This is a key pillar of the pandemic response in Toronto, Ontario, a city of about 3 million people. In mid-March 2020, the Ontario government developed COVID-19 assessment centres throughout the province to facilitate testing. These assessment centres provide SARSCoV-2 nasopharyngeal swab testing to the public and health care workers based on algorithms that consider symptoms, known or suspected exposure to SARS-CoV-2, travel history and involvement with vulnerable at-risk populations.

We sought to characterize the diagnostic test characteristics (i.e., sensitivity, specificity, and positive and negative predictive values) of anosmia as an early symptom of SARSCoV-2 infection in an outpatient population with varying degrees of risk and symptoms of COVID-19. We also planned to describe the demographic characteristics and clinical presentations of people who tested positive for SARSCoV-2 infection.

\section{Methods}

\section{Study design}

We conducted a retrospective, repeated, cross-sectional (chart review) study including consecutive patients undergoing a SARS-CoV-2 test at 2 COVID-19 assessment centres in Toronto from Apr. 5 to Sept. 30, 2020. This study included data from COVID-19 assessment centres affiliated with 2 academic hospitals in Toronto. North York General Hospital (NYGH) is a medium-sized community hospital with 435 inpatient beds; data in this study are from the hospital's COVID-19 assessment centre in an outpatient setting in a lower-income neighbourhood in northwestern Toronto. ${ }^{16}$ Women's College Hospital (WCH) is an outpatient hospital in the downtown core, with a focus on women's health, but its COVID-19 assessment centre provides testing to people of all genders and ages. ${ }^{17} \mathrm{We}$ applied the checklist for The Reporting of Studies Conducted Using Observational Routinely Collected Health Data (RECORD) guidelines for this observational study. ${ }^{18}$

\section{Population}

Access to the centres during the study period (Apr. 5Sept. 30, 2020) was either by walk-in or online self-assessment for initial screening to determine eligibility. At the time of registration, health care providers asked people questions about symptoms and documented responses into the hospital electronic health records (EHRs). All data included in this study were from patient self-report at the time of registration at the clinic and from SARS-CoV-2 test results obtained from samples collected at these 2 testing clinics.

Both symptomatic and asymptomatic people were tested on the basis of evolving screening criteria from local public health authorities. When COVID-19 assessment centres opened on Mar. 12, 2020, only symptomatic individuals could be tested. This changed on May 28, 2020, when asymptomatic people who were concerned about COVID-19 could be tested. ${ }^{19}$ The criteria then reverted back to testing only symptomatic individuals as of Sept. 24, 2020. ${ }^{20}$ Individuals accessing the testing sites had varying degrees of risks of contracting SARS-CoV-2 infection, ranging from those with confirmed or suspected close contact with someone infected with SARS$\mathrm{CoV}-2$, to asymptomatic people requesting testing required before surgical procedures or visits to relatives in long-term care facilities, or for reassurance.

The centres operated 7 days per week and tested between 100 and 2000 patients weekly as the pandemic progressed. Testing for SARS-CoV-2 used reverse transcription polymerase chain reaction (RT-PCR) with gene sequencing for nucleocapsid, envelope and RNA-dependent RNA polymerase (RdRp; enzyme). ${ }^{21,22}$

\section{Data collection}

Clinical data from the COVID-19 assessment centres' flowsheets were abstracted (S.K.) from the Cerner and Epic EHRs at NYGH and WCH, respectively, and exported into Microsoft Excel spreadsheets. Common data variables collected from both hospitals included age, sex, postal code, exposure history (i.e., travel outside of Canada within 14 days, contact with confirmed or suspected case of COVID-19, or health care worker), vital signs (i.e., heart rate, blood pressure, oxygen saturation and temperature) and symptoms (e.g., anosmia, cough and/or shortness of breath, diarrhea and/or abdominal pain, and fever). A question about altered or diminished sense of smell was adapted from Hoffman and colleagues, ${ }^{23}$ as follows: "Have you had a new problem with your ability to smell, such as not being able to smell things or things not smelling the way they are supposed to?" This approach has been reported as having moderate sensitivity for anosmia $(54.4 \%)$ and severe hyposmia $(78.1 \%) .^{24}$

SARS-CoV-2 test results were coded as negative or positive. Any subsequent PCR tests done after a positive result, which were conducted early in the pandemic to assess for virus clearance, were excluded, since they would not be new infections and would result in duplicate data for positive cases.

The WCH electronic record data contained additional variables that we extracted, including change in taste, cough, difficulty swallowing, fatigue, headache, hoarse voice, myalgia, nasal congestion, nausea, vomiting, respiratory distress, runny nose, sneezing and sore throat.

During the data extraction process, we verified the accuracy of EHR data using a manual chart abstraction for a random subset of 100 patients and did not find erroneous information. The data elements collected at both COVID assessment centres are further described in Appendix 1 (available at www. cmajopen.ca/content/9/4/E1134/suppl/DC1). 


\section{Statistical analysis}

Deidentified data from each institution's Microsoft Excel spreadsheet were combined and exported into SAS version 9.4 (SAS Corp.) for statistical analysis. We conducted primary analyses on the combined data of common variables, as well as secondary analyses within each cohort, including sitespecific variables.

We calculated diagnostic test characteristics (sensitivity, specificity, positive predictive value [PPV], negative predictive value [NPV], accuracy [true negative + true positive / true negative + true positive + false negative + false positive], likelihood ratio + [LR+], and likelihood ratio - [LR-]) for the onset of anosmia in predicting SARS-CoV-2 infection status in people presenting to the assessment centres. ${ }^{25}$ Diagnostic measures were also calculated for common symptoms at both assessment centres and for additional symptoms collected at WCH separately.

We used generalized estimating equations with exchangeable correlation structure for the binary outcome of SARS$\mathrm{CoV}-2$ results (positive/negative) to capture the patient-level dependence for repeat COVID-19 screening during the study period. ${ }^{26}$ Generalized estimating equations were also used to adjust for participant demographic characteristics (age, sex and travel history) and common symptoms available at both sites (anosmia, cough and/or shortness of breath, and diarrhea and/or abdominal pain).

\section{Ethics approval}

This study was approved by NYGH's and WCH's research ethics boards (protocol nos. 20-0021 and 2020-0059-E). These approvals included permission to waive written informed consent given that this study was conducted using routinely collected health information.

\section{Results}

A total of 83443 tests were completed (53479 at NYGH [Apr. 12-Sept. 30] and 29964 at WCH [Apr. 5-Sept. 30]) for 72692 participants. Participants' demographic characteristics and reported symptoms are summarized in Table 1 . The overall test positivity rate was $2.0 \%(2.3 \%$ at NYGH and $1.4 \%$ at $\mathrm{WCH}$ ). The positivity rate was $2.3 \%$ among adults aged 20-29 years, $1.5 \%$ among those younger than 20 years, and $1.3 \%$ among adults aged 60 years and older.

The prevalence of a positive SARS-CoV-2 test result was higher in those reporting anosmia than in those not reporting anosmia $(12.0 \%$ v. $1.7 \%)$. Test characteristics for the association between anosmia and SARS-CoV-2 positivity were as follows: sensitivity 0.138 (95\% CI $0.121-0.154$ ), specificity 0.980 (95\% CI 0.979-0.981), PPV 0.120 (95\% CI $0.106-0.135)$, NPV 0.983 (95\% CI $0.982-0.984$ ) and accuracy 0.963 (95\% CI $0.962-0.965)$ (Figure 1 ).

The prevalence of positive SARS-CoV-2 test results was higher among patients with clinical symptoms including fever (7.0\% v. $1.6 \%)$, cough and/or shortness of breath $(5.1 \% \mathrm{v}$. $1.5 \%)$, and diarrhea and/or abdominal pain (2.9\% v. $1.9 \%)$.

Low sensitivity and high specificity with low PPV and high NPV were observed for common symptoms recorded at both assessment centres (Figure 1; Appendix 2, available at www. cmajopen.ca/content/9/4/E1134/supp1/DC1).

At WCH, where additional symptoms were captured (Figure 2; Appendix 3, available at www.cmajopen.ca/content/9/4/ E1134/suppl/DC1), the highest sensitivity was observed for cough $(0.450,95 \%$ CI $0.405-0.496)$, headache $(0.318,95 \%$ CI $0.275-0.361)$ and fatigue $(0.276,95 \%$ CI $0.235-0.317)$, while symptoms with the highest specificity included anosmia $(0.987$, 95\% CI $0.986-0.988)$, change in taste $(0.984,95 \%$ CI $0.983-$ $0.986)$ and difficulty swallowing $(0.975,95 \%$ CI $0.973-0.977)$.

Out of 1640 patients across both sites who had positive SARS-CoV-2 tests, 837 (51.0\%) were asymptomatic and reported no anosmia, cough, shortness of breath, fever, diarrhea or abdominal pain.

Figure 3 (and Appendix 4, Table S4, available at www. cmajopen.ca/content/9/4/E1134/suppl/DC1) shows the crude and adjusted odds ratios (ORs) for SARS-CoV-2 test positivity with respect to age, sex and common symptoms at both testing sites. With the exception of diarrhea and/or abdominal pain (adjusted OR $0.71,95 \%$ CI $0.60-0.85$ ), patients with any symptoms who presented to the clinic had increased odds of a positive SARS-CoV-2 test. The adjusted odds ratio for a positive SARS-CoV-2 test was 5.29 (95\% CI 4.50-6.22).

\section{Interpretation}

In our retrospective, repeated, cross-sectional study of 83443 SARS-CoV-2 tests in a community-based sample, we identified a moderate association between self-reported anosmia and positive SARS-CoV-2 test results.

Our findings are consistent with the results of a metaanalysis on the association between anosmia and SARS-CoV-2 test positivity that reported an OR of $14.7 .{ }^{10}$ While the adjusted OR reported in our study was lower at 5.29, this may reflect that, throughout our study period, there were ongoing changes in testing criteria, and most people who reported COVID-19like symptoms were from areas with relatively low prevalence.

Our identified association between anosmia and SARS$\mathrm{CoV}-2$ positivity is also lower than what was identified in another Toronto study in which people were retrospectively contacted to ask about the presence of this symptom; ${ }^{11}$ we believe our findings more accurately reflect the true prevalence of anosmia associated with SARS-CoV-2 infection at the time patients present, with a lower risk of recall bias.

Anosmia is a common symptom of other conditions, such as allergic rhinitis $(21 \%)^{27}$ and other upper respiratory tract infections (30\%). ${ }^{28}$ In our study, anosmia was present among $12 \%$ of people who tested positive for SARS-CoV-2 infection; more importantly, anosmia had very high specificity $(98 \%)$. However, because of the low prevalence of SARS$\mathrm{CoV}-2$ infection in these settings (overall test positivity was $2 \%)$, the positive predictive value was low (12\%). While anosmia had the strongest association with SARS-CoV-2 positivity among people in the sample, the test characteristics make its clinical usefulness limited to raising suspicion of this diagnosis, rather than strongly suggesting it. Given that PPV varies by prevalence, small changes in prevalence 
Table 1 (part 1 of 2): Demographic characteristics and symptoms of people who underwent a SARS-CoV-2 test at COVID-19 assessment centres at NYGH and WCH

SARS-CoV-2 swab test; no. $(\%)^{*}$

\begin{tabular}{ll}
\multicolumn{2}{c}{$\begin{array}{c}\text { SARS-CoV-2 swab test; } \\
\text { no. }(\%)^{*}\end{array}$} \\
\cline { 2 - 3 } Variable & Negative $\quad$ Positive no.
\end{tabular}

\begin{tabular}{l|}
\hline Demographic characteristics \\
\hline Site
\end{tabular}

\begin{tabular}{|llll}
\hline NYGH (site 1) & $52272(97.7)$ & $1207(2.3)$ & 53479 \\
\hline WCH (site 2) & $29531(98.6)$ & $433(1.4)$ & 29964 \\
\hline
\end{tabular}

Age group, yr

\begin{tabular}{|lccc|}
\hline $0-9$ & $4563(99.0)$ & $46(1.0)$ & 4609 \\
\hline $10-19$ & $5093(98.1)$ & $98(1.9)$ & 5191 \\
\hline $20-29$ & $18488(97.8)$ & $425(2.2)$ & 18913 \\
\hline $30-39$ & $16602(98.2)$ & $312(1.8)$ & 16914 \\
\hline $40-49$ & $11617(97.4)$ & $306(2.6)$ & 11923 \\
\hline $50-59$ & $11446(97.8)$ & $262(2.2)$ & 11708 \\
\hline $60-69$ & $8570(98.4)$ & $140(1.6)$ & 8710 \\
\hline$\geq 70$ & $5419(99.1)$ & $51(0.9)$ & 5470 \\
\hline Missing & $5(100.0)$ & & 5 \\
\hline Sex & & & \\
\hline
\end{tabular}

\begin{tabular}{|cccc|}
\hline Female & $47646(98.1)$ & $918(1.9)$ & 48564 \\
\hline Male & $34157(97.9)$ & $722(2.1)$ & 34879 \\
\hline Travel† & & & \\
\hline No & $80090(98.1)$ & $1586(1.9)$ & 81676 \\
\hline Yes & $1713(96.9)$ & $54(3.1)$ & 1767 \\
\hline
\end{tabular}

\section{Symptoms}

Anosmia

\begin{tabular}{|cccc|}
\hline No & $80152(98.3)$ & $1414(1.7)$ & 81566 \\
\hline Yes & $1651(88.0)$ & $226(12.0)$ & 1877 \\
\hline \multicolumn{1}{|c|}{ Cough and/or shortness of breath } & & \\
\hline No & $71329(98.5)$ & $1076(1.5)$ & 72405 \\
\hline Yes & $10474(94.9)$ & $564(5.1)$ & 11038 \\
\hline Fever & & \\
\hline No & $76370(98.4)$ & $1232(1.6)$ & 77602 \\
\hline Yes & $5433(93.0)$ & $408(7.0)$ & 5841 \\
\hline Diarrhea and/or abdominal pain & & \\
\hline No & $76225(98.1)$ & $1474(1.9)$ & 77699 \\
\hline Yes & $5578(97.1)$ & $166(2.9)$ & 5744 \\
\hline Pulse rate, beats/min & & \\
\hline Missing & $56657(98.7)$ & $761(1.3)$ & 57418 \\
\hline $20-60$ & $1470(97.4)$ & $39(2.6)$ & 1509 \\
\hline $61-90$ & $19006(96.9)$ & $618(3.1)$ & 19624 \\
\hline$\geq 91$ & $4670(95.5)$ & $222(4.5)$ & 4892 \\
\hline
\end{tabular}

Table 1 (part 2 of 2): Demographic characteristics and symptoms of people who underwent a SARS-CoV-2 test at COVID-19 assessment centres at NYGH and WCH

\begin{tabular}{|c|c|c|c|}
\hline \multirow[b]{2}{*}{ Variable } & \multicolumn{2}{|c|}{$\begin{array}{c}\text { SARS-CoV-2 swab test; } \\
\text { no. }(\%)^{*}\end{array}$} & \multirow[b]{2}{*}{ Total, no. } \\
\hline & Negative & Positive & \\
\hline \multicolumn{4}{|l|}{ Symptoms cont'd } \\
\hline \multicolumn{4}{|l|}{ Body temperature } \\
\hline Missing & $51558(99.0)$ & $496(0.9)$ & 52054 \\
\hline $\begin{array}{l}93-97.9^{\circ} \mathrm{F} \\
\left(33.9-36.6^{\circ} \mathrm{C}\right)\end{array}$ & $21711(96.8)$ & 717 (3.2) & 22428 \\
\hline $\begin{array}{l}98-98.9^{\circ} \mathrm{F} \\
\left(36.7-37.2^{\circ} \mathrm{C}\right)\end{array}$ & $7474(96.1)$ & 307 (3.9) & 7781 \\
\hline $\begin{array}{l}\geq 99^{\circ} \mathrm{F} \\
\left(\geq 37.3^{\circ} \mathrm{C}\right)\end{array}$ & $1060(89.8)$ & $120(10.2)$ & 1180 \\
\hline \multicolumn{4}{|c|}{ Respiratory rate, breaths/min } \\
\hline Missing & 55707 (98.8) & $666(1.2)$ & 56373 \\
\hline$\leq 24$ & $25856(96.4)$ & $964(3.6)$ & 26820 \\
\hline$>24$ & $240(96.0)$ & $10(4.0)$ & 250 \\
\hline \multicolumn{4}{|c|}{ Systolic blood pressure, $\mathrm{mm} \mathrm{Hg}$} \\
\hline Missing & $59368(99.0)$ & $624(1.0)$ & 59992 \\
\hline$\leq 110$ & $2596(96.1)$ & $104(3.8)$ & 2700 \\
\hline$>110$ & $19839(95.6)$ & $912(4.4)$ & 20751 \\
\hline \multicolumn{4}{|c|}{ Saturated oxygen, \% } \\
\hline Missing & $51204(99.0)$ & $495(1.0)$ & 51699 \\
\hline$\leq 92$ & $62(89.9)$ & $7(10.1)$ & 69 \\
\hline$>92$ & $30537(96.4)$ & $1138(3.6)$ & 31675 \\
\hline Total & $81803(98.0)$ & $1640(2.0)$ & 83443 \\
\hline \multicolumn{4}{|c|}{$\begin{array}{l}\text { Note: } \mathrm{NYGH}=\text { North York General Hospital, } \mathrm{WCH}=\text { Women's College Hospital. } \\
\text { *This is a row percentage. } \\
\text { tTravel outside Canada within last } 14 \text { days (relative to screening test date). }\end{array}$} \\
\hline
\end{tabular}

increase PPV substantially: for example, with a $10 \%$ prevalence, the PPV would be $43 \%$. No other symptom was sufficiently associated with SARS-CoV-2 positivity to suggest its use for screening or for predicting a positive test.

\section{Limitations}

This study was completed in 2020, and symptomatology related to newer SARS-CoV-2 variants may differ. Other limitations of this study include the possibility of falsenegative and false-positive tests, as well as the heterogenous study population. Additional data were available from the WCH assessment centre because no provincial standard existed for what data should be collected at COVID-19 assessment centres, and each organization designed its own data collection independently. This study is from a single city in one province and may not be generalizable to other settings, particularly related to varying incidence of COVID-19 between settings. 


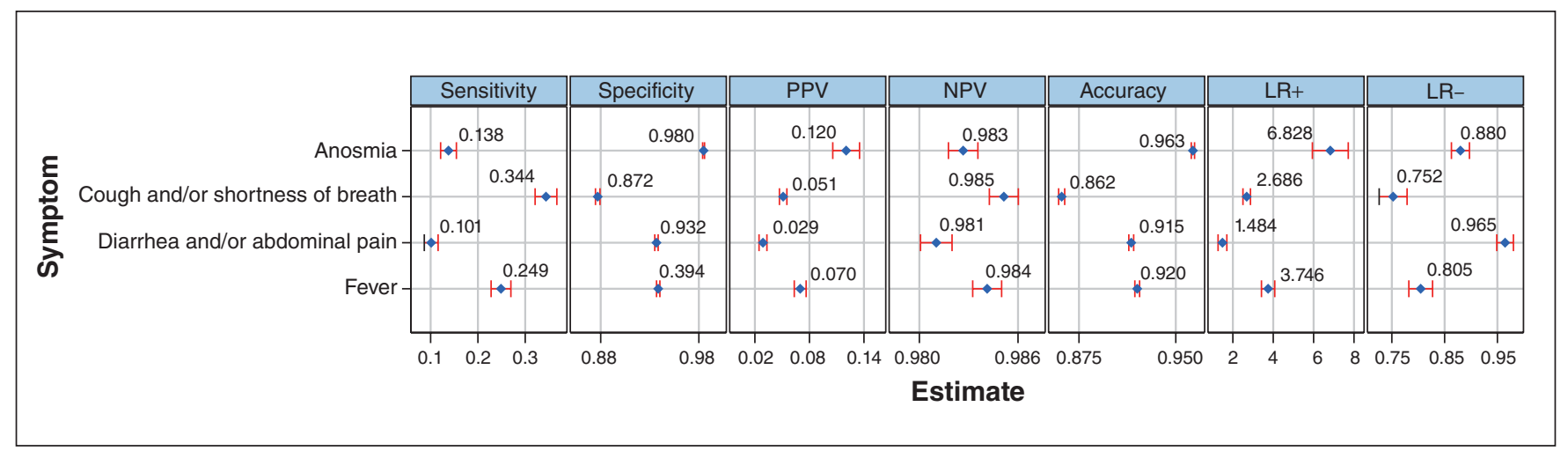

Figure 1: Diagnostic measures of COVID-19 symptoms recorded at North York General Hospital and Women's College Hospital (point estimates in blue, $95 \%$ confidence intervals in red). Note: $L R=$ likelihood ratio, NPV = negative predictive value, PPV = positive predictive value.

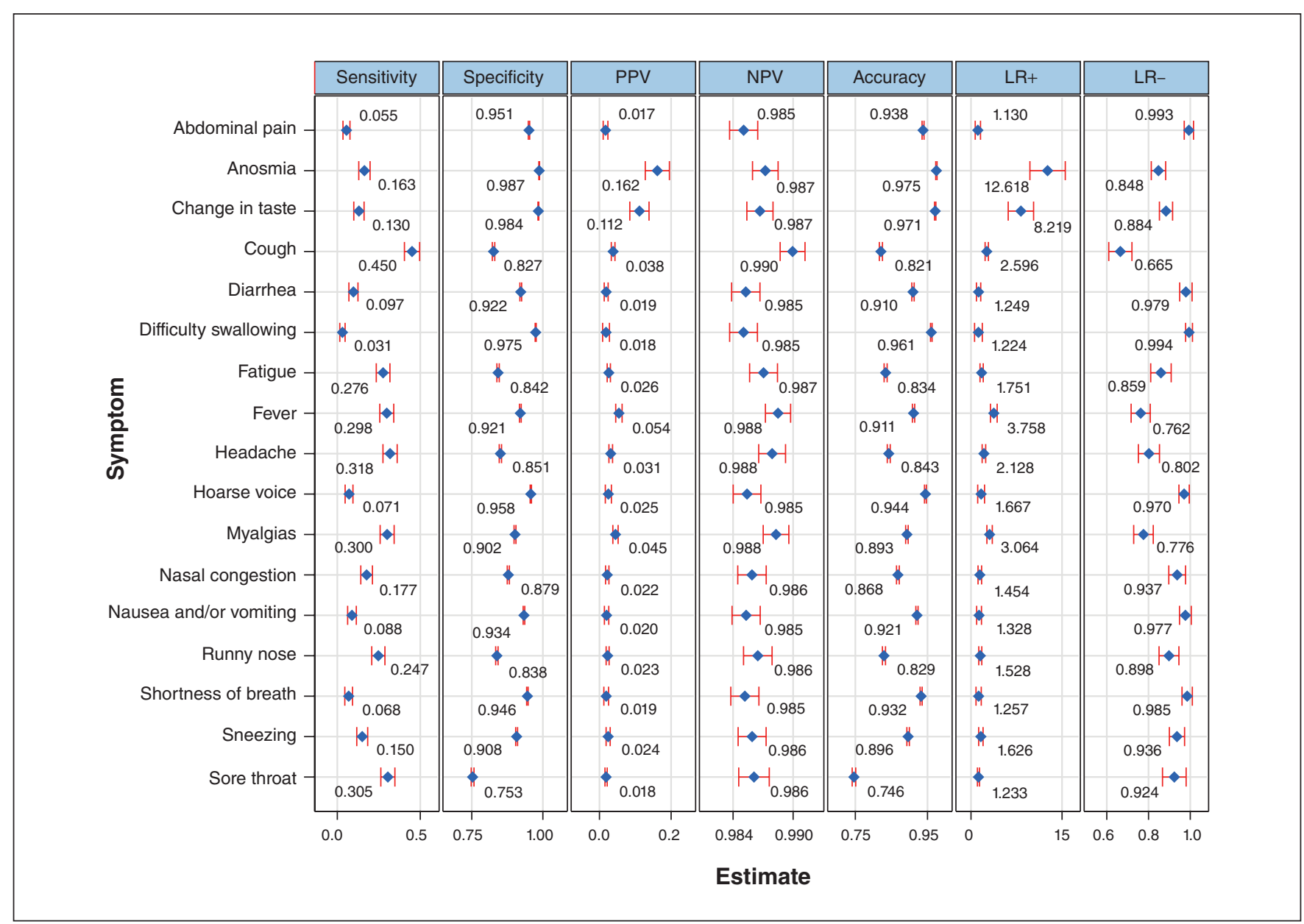

Figure 2: Diagnostic measures of COVID-19 symptoms recorded at Women's College Hospital (point estimates in blue, 95\% confidence intervals in red). Note: $L R=$ likelihood ratio, NPV = negative predictive value, $\mathrm{PPV}=$ positive predictive value.

SARS-CoV-2 testing was conducted for various indications throughout the study period, as knowledge about the disease and testing capacity changed over time. The purpose of tests also varied, from screening asymptomatic people before outpatient procedures, to diagnostic testing for people with symptoms thought to be consistent with
COVID-19 or who had a high risk for SARS-CoV-2 infection. This study includes data from anyone who was tested, and we were unable to analyze data by testing indication. A recent systematic review identified 2 studies reporting $20 \%$ and $75 \%$ prevalence of asymptomatic people among those testing positive. ${ }^{4}$ 


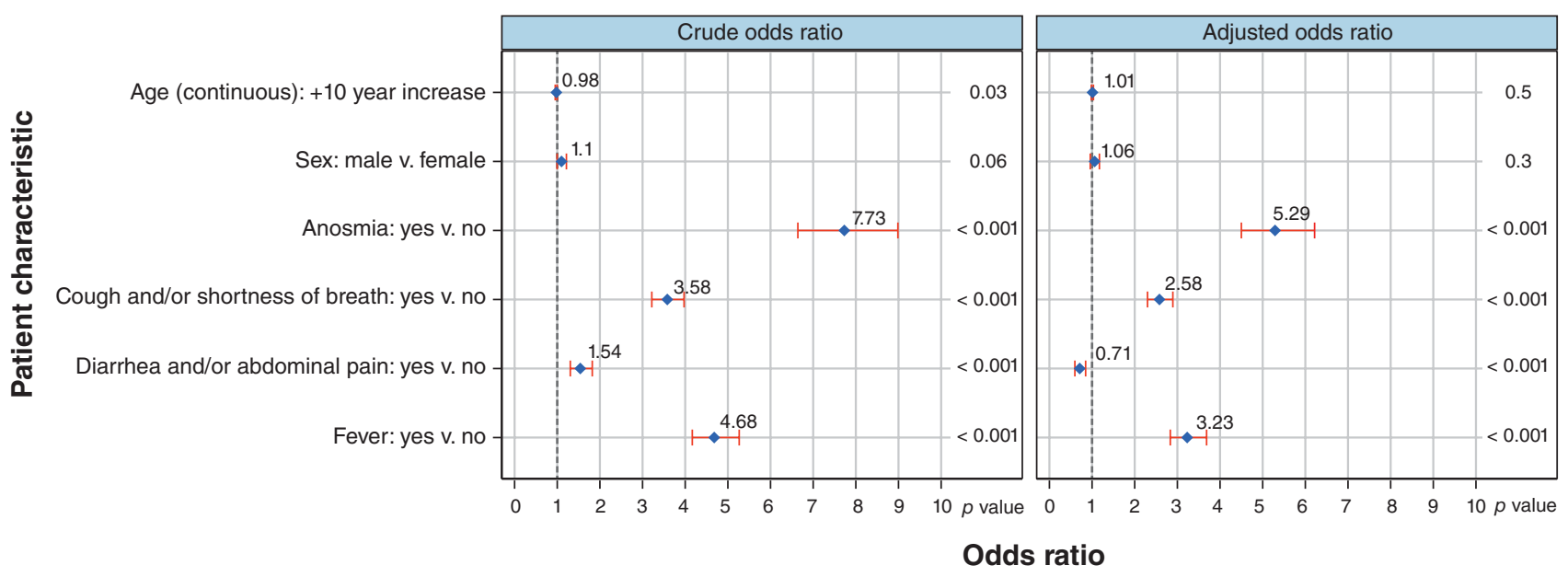

Figure 3: Odds ratios (ORs) for positive SARS-CoV-2 swab test, by patient characteristic (95\% confidence intervals in red, dotted line shows $\mathrm{OR}=1$; results adjusted for age, sex, travel history and common symptoms available at both study sites: anosmia, cough and/or shortness of breath, diarrhea and/or abdominal pain).

Our approach to assessing anosmia was identified through a brief literature review and represented the most feasible approach we could identify at the time that would be possible to complete in these clinical contexts. Anosmia could have been underestimated given the $54 \%-78 \%$ sensitivity of the "single question" approach used here ${ }^{23}$ when compared with more intensive testing for anosmia, such as the Mini Olfactory Questionnaire approach ${ }^{29}$ used in another Canadian study reporting an association between anosmia and positive SARSCoV-2 tests. ${ }^{7}$ Data in our study were self-reported and may be subject to recall bias or underestimation for that reason as well: an Iranian study from April 2020 using objective measurement identified a $98 \%$ prevalence of hyposmia or anosmia among 60 inpatients with positive SARS-CoV-2 tests, which was higher than our identified prevalence. ${ }^{30}$

Patients were asked about anosmia using the question as described in the Methods section ("Have you had a new problem with your ability to smell, such as not being able to smell things or things not smelling the way they are supposed to?") without additional details. There was no specific guidance given about what "new" meant; it is possible if people asked for clarification about this, health care providers asking the questions provided their own interpretation. The question was piloted for a few days along with other data elements before it was incorporated into systematic data collection as part of starting up the clinics, and we did not receive any feedback about patients requesting clarification. It is unknown how this might have affected the diagnostic test characteristics of the questions.

Finally, we generated the statistical inference using completecase analysis in which associations were captured using recorded clinical characteristics. It is necessary to acknowledge this limitation, as the observational EHR data may be prone to different sources of missingness (e.g., missing at random, missing not at random) and this, in turn, may lead to results with less generalizability.

\section{Conclusion}

In this study involving people attending 2 community-based COVID-19 assessment centres, presence of anosmia did not reliably identify participants with SARS-CoV-2 infection. However, anosmia's high specificity and positive predictive value of $12 \%$ in this community population with low prevalence of SARS-CoV-2 positivity suggests a moderate clinical suspicion of infection in individuals with this symptom. This finding suggests that people with new-onset anosmia should consider being tested for SARS-CoV-2.

\section{References}

1. COVID-19 coronavirus pandemic. Worldometer. Available: https://www worldometers.info/coronavirus/ (accessed 2021 Nov. 18).

2. Coronavirus. Geneva: World Health Organization. Available: https://www. who.int/health-topics/coronavirus\#tab=tab_1 (accessed 2020 Apr. 1).

3. Wynants L, Van Calster B, Collins GS, et al. Prediction models for diagnosis and prognosis of covid-19: systematic review and critical appraisal. $B M 7$ 2020;369:m1328.

4. Yanes-Lane M, Winters N, Fregonese F, et al. Proportion of asymptomatic infection among COVID-19 positive persons and their transmission potential: a systematic review and meta-analysis. PLoS One 2020;15:e0241536.

5. Loss of sense of smell as marker of COVID-19 infection. London (UK): ENT UK at the Royal College of Surgeons of England. Available: https:// www.entuk.org/sites/default/files/files/Loss \%20of\%20sense \%20of\%20smell \%20as\%20marker\%20of\%20COVID.pdf (accessed 2021 Mar. 11).

6. Roberts M. Coronavirus: Are loss of smell and taste key symptoms? $B B C$ News 2020 Apr. 1. Available: https://www.bbc.com/news/health-52111606 (accessed 2021 Mar. 11).

7. Carignan A, Valiquette L, Grenier C, et al. Anosmia and dysgeusia associated with SARS-CoV-2 infection: an age-matched case-control study. CMAf 2020;192:E702-7.

8. Bénézit F, Le Turnier P, Declerck C, et al.; RAN COVID Study Group. Utility of hyposmia and hypogeusia for the diagnosis of COVID-19. Lancet Infect Dis 2020;20:1014-5.

9. Pang KW, Chee J, Subramaniam S, et al. Frequency and clinical utility of olfactory dysfunction in COVID-19: a systematic review and meta-analysis. Curr Allergy Asthma Rep 2020;20:76.

10. Aziz M, Goyal H, Haghbin H, et al. The association of "loss of smell" to COVID-19: a systematic review and meta-analysis. Am 7 Med Sci 2021;361: 216-25.

11. Lee DJ, Lockwood J, Das P, et al. Self-reported anosmia and dysgeusia as key symptoms of COVID-19. CFEM 2020;22:595-602.

12. Struyf T, Deeks JJ, Dinnes J, et al.; Cochrane COVID-19 Diagnostic Test Accuracy Group. Signs and symptoms to determine if a patient presenting in 
primary care or hospital outpatient settings has COVID-19. Cochrane Database Syst Rev 2021;2:CD013665.

13. Boesveldt S, Postma EM, Boak D, et al. Anosmia: a clinical review. Chem Senses 2017; 42:513-23.

14. Jaume F, Quintó L, Alobid I, et al. Overuse of diagnostic tools and medications in acute rhinosinusitis in Spain: a population-based study (the PROSINUS study). BM7 Open 2018;8:e018788.

15. Matukas LM, Dhalla IA, Laupacis A. Aggressively find, test, trace and isolate to beat COVID-19. CMA7 2020;192:E1164-5.

16. Statistics and area served. Toronto: North York General Hospital; 2019. Available: https://www.nygh.on.ca/about-us/overview/statistics-and-area-served (accessed 2021 July 16)

17. Who we are. Toronto: Women's College Hospital. Available: https://www. womenscollegehospital.ca/about-wch/who-we-are/ 2021 July 16).

18. Nicholls SG, Quach P, von Elm E, et al. The REporting of studies Conducted using Observational Routinely-collected health Data (RECORD) statement: methods for arriving at consensus and developing reporting guidelines. PLoS One 2015;10:e0125620.

19. Recommendations for COVID-19 assessment centres. Toronto: Ontario Health; 2020 May 28, updated 2021 July 7. Available: https://www.ontario health.ca/sites/ontariohealth/files/2021-07/AssessmentCentres.pdf (accessed 2021 July 16).

20. Ontario updates COVID-19 testing guidelines [news release]. Toronto: Ontario Ministry of Health; 2020 Sept. 24. Available: https://news.ontario.ca/ en/statement/58507/ontario-updates-covid-19-testing (accessed 2021 July 16).

21. Coronavirus disease 2019 (COVID-19): PCR. Public Health Ontario. 16 March 2020. Available: https://www.publichealthontario.ca/en/laboratory-services/test -information-index/wuhan-novel-coronavirus (accessed 2020 Apr. 1).

22. Corman V, Bleicker T, Brünink S, et al. Diagnostic detection of 2019-nCoV by real-time RT-PCR. Geneva: World Health Organization; 2020. Available: https:/www.who.int/docs/default-source/coronaviruse/protocol-v2-1.pdf?sfursn =a9ef618c_2 (accessed 2020 Apr. 1).

23. Hoffman HJ, Ishii EK, Macturk RH. Age-related changes in the prevalence of smell/taste problems among the United States adult population: results of the 1994 disability supplement to the National Health Interview Survey (NHIS). Ann N Y Acad Sci 1998;855:716-22.

24. Hoffman HJ, Rawal S, Li C-M, et al. New chemosensory component in the US National Health and Nutrition Examination Survey (NHANES): first-year results for measured olfactory dysfunction. Rev Endocr Metab Disord 2016; 17:221-40.

25. Pepe MS. The statistical evaluation of medical tests for classification and prediction. Oxford (UK): Oxford University Press; 2003.

26. Zeger SL, Liang KY, Albert PS. Models for longitudinal data: a generalized estimating equation approach. Biometrics 1988;44:1049-60.

27. Rydzewski B, Pruszewicz A, Sulkowski WJ. Assessment of smell and taste in patients with allergic rhinitis. Acta Otolaryngol 2000;120:323-6.

28. Seiden AM. Postviral olfactory loss. Otolaryngol Clin North Am 2004;37:1159-66.

29. Zou L-Q, Linden L, Cuevas M, et al. Self-reported mini olfactory questionnaire (Self-MOQ): a simple and useful measurement for the screening of olfactory dysfunction. Laryngoscope 2020;130:E786-90.

30. Moein ST, Hashemian SM, Mansourafshar B, et al. Smell dysfunction: a biomarker for COVID-19. Int Forum Allergy Rhinol 2020;10:944-50.

Affiliations: MAP Centre for Urban Health Solutions (O’Neill, Pinto), Li Ka Shing Knowledge Institute; Department of Family and Community Medicine (O'Neill, Pinto), St. Michael's Hospital, Unity Health; Department of Family and Community Medicine (O'Neill, Kalia, Greiver, Agarwal, Eisen, Pinto, Dunn), Temerty Faculty of Medicine, University of Toronto; SickKids Research Institute (Gill), Child Health Evaluative Sciences, Peter Gilgan Centre for Research and Learning; Department of Family and Community Medicine (Hum, Agarwal,
Dunn), Women's College Hospital; North York General Hospital (Moran-Venegas); Department of Family and Community Medicine (Stoller, Greiver, Eisen), North York General Hospital; Postgraduate Medical Education (Kirubarajan, DeKoven), Temerty Faculty of Medicine, University of Toronto, Toronto, Ont.

Contributors: Braden O'Neill and Sheila Dunn developed the initial study idea. Peter Gill, Samuel DeKoven, Susan Hum, Michelle Greiver, Payal Agarwal, Abirami Kirubarajan, Sheila Dunn and Andrew Pinto contributed substantively to the study design and overall approach. Carla Moran-Venegas, Rebecca Stoller, Susan Hum and David Eisen facilitated data access and contributed to the analysis plan. Sumeet Kalia conducted the analysis. Braden O'Neill wrote the first draft of the manuscript, and all authors provided substantive comments on the manuscript over several iterations. All authors gave final approval of the version to be published and agreed to be accountable for all aspects of the work.

Funding: Support for data extraction and analysis was provided in-kind by the University of Toronto Practice-Based Research Network (UTOPIAN), North York General Hospital and Women's College Hospital. Braden O'Neill and Andrew Pinto are supported as clinician scientists by the Department of Family and Community Medicine, Temerty Faculty of Medicine, University of Toronto, the Department of Family and Community Medicine, St. Michael's Hospital, and the Li Ka Shing Knowledge Institute, St. Michael's Hospital. Andrew Pinto is also supported by a fellowship from the Physicians' Services Incorporated Foundation and as the associate director for clinical research at UTOPIAN. Michelle Greiver is the director of UTOPIAN and the Gordon F. Cheesbrough Chair in Family and Community Medicine, North York General Hospital, Toronto. Sheila Dunn is supported as a clinician investigator by the Department of Family and Community Medicine, Temerty Faculty of Medicine, University of Toronto, and as a scientist by the Women's College Research Institute and the Department of Family and Community Medicine, Women's College Hospital, Toronto.

Content licence: This is an Open Access article distributed in accordance with the terms of the Creative Commons Attribution (CC BY-NC-ND 4.0) licence, which permits use, distribution and reproduction in any medium, provided that the original publication is properly cited, the use is noncommercial (i.e., research or educational use), and no modifications or adaptations are made. See: https://creativecommons.org/licenses/by-nc-nd/4.0/

Data sharing: The data included in this study are not publicly available; analytic code is available on request from the corresponding author. Requests to conduct additional analyses should be directed to the corresponding author.

Acknowledgement: The authors thank everyone who worked in the North York General and Women's College Hospital COVID-19 assessment centres.

Disclaimer: Braden O'Neill is an associate editor for CMA7 and CMAf Open, and Peter Gill is a member of CMAJ Open's editorial board. They were not involved in the editorial decision-making process for this article.

Supplemental information: For reviewer comments and the original submission of this manuscript, please see www.cmajopen.ca/content/9/4/ E1134/suppl/DC1. 